\title{
Design Study "Lite" Methodology: Expediting Design Studies and Enabling the Synergy of Visualization Pedagogy and Social Good
}

\author{
Uzma Haque Syeda \\ Northeastern University \\ syeda.u@northeastern.edu
}

\author{
Prasanth Murali \\ Northeastern University \\ murali.pr@northeastern.edu
}

\author{
Lisa Roe \\ Northeastern University \\ 1.roe@northeastern.edu
}

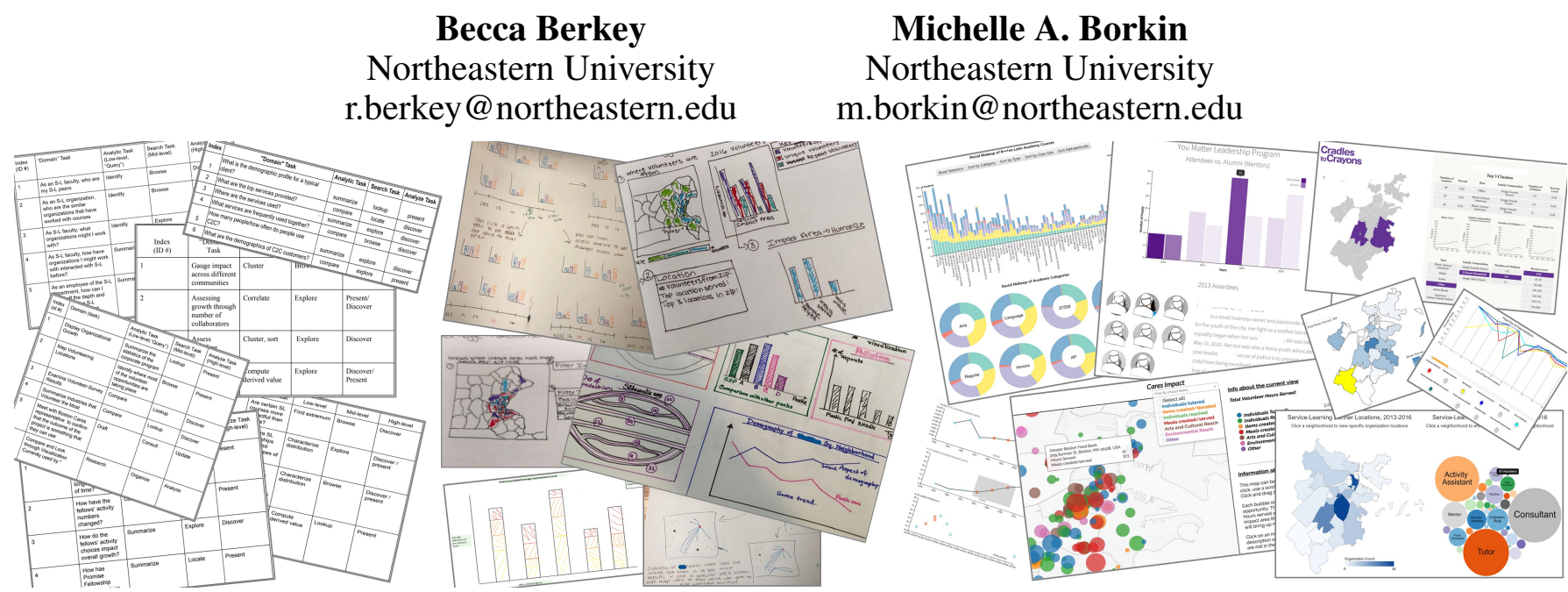

Figure 1. The figure presents examples of how students implemented the Design Study "Lite" Methodology. Task abstractions, ideation process low-fidelity sketches, and final visualizations are shown in the piles from left to right respectively.

\section{ABSTRACT}

Design studies are frequently used to conduct problem-driven visualization research by working with real-world domain experts. In visualization pedagogy, design studies are often introduced but rarely practiced due to their large time requirements. This limits students to a classroom curriculum, often involving projects that may not have implications beyond the classroom. Thus we present the Design Study "Lite" Methodology, a novel framework for implementing design studies with novice students in 14 weeks. We utilized the Design Study "Lite" Methodology in conjunction with Service-Learning to teach five Data Visualization courses and demonstrate that it benefits not only the students but also the community through service to non-profit partners. In this paper, we provide a detailed breakdown of the methodology and how Service-Learning can be incorporated with it. We also include an extensive reflection on the methodology and provide recommendations for future applications of the framework for teaching visualization courses and research.
Author Keywords

Visualization, Design Studies, Service-Learning, Pedagogy, Theory and Methods

\section{CCS Concepts}

-Human-centered computing $\rightarrow$ Visualization; Information visualization; Visualization theory, concepts and paradigms; •Applied computing $\rightarrow$ Education;

\section{INTRODUCTION}

Design studies are a form of problem-driven research in which visualization researchers investigate and solve a real-world problem faced by domain experts through visualization of their data. The field of visualization requires a synergy between technique-driven and problem-driven research [1] in order to maintain vitality, thus underlying the importance of design studies to the community. In fact, design study papers are one of the five categories of publications in the IEEE VIS conference. Although researched as a theoretical concept and executed in practice, design studies are typically not included in visualization pedagogy. In reviewing the course websites of the 2019 VAST Program, InfoVis Program, and InfoVis Steering Committee members (139 in total with some overlaps) and narrowing down the list of accessible courses (29), we found that $89 \%$ of the courses, do not have practical implementation of design studies within their curriculum, allowing a gap between budding visualization students and researchers. 
Teaching design studies to students is very challenging due to the long timelines required to execute such studies. Typical design studies take several months to even years for completion $[1,2,3,4]$. This is due to the need to become familiar with the domain, possible limited availability of the domain expert(s), and an iterative process of data analysis, visualization design, and implementation. Moreover, design studies are hard to do well and can fall into many potential pitfalls [1]. Hence, to teach and execute design studies that fit the duration of a typical semester, modifications and specifications to the design study methodology are necessary that make it easier to understand and apply in the requisite domain.

In this paper, we present the Design Study "Lite" Methodology ("DSLM") which adapts the nine-stage Design Study Methodology proposed by Sedlmair et al. [1] and expedites the design study process to fit within the time frame of 14 weeks. DSLM was first proposed in a workshop paper at the Pedagogy of Data Visualization Workshop (PDVW) at IEEE VIS 2017 [2]. Our work is a detailed extension of this prior work with over 2 years of iterative development and improvement of the methodology for executing design study projects in undergraduate and graduate visualization courses.

Meyer et al. [5] proposes methodologies for ensuring rigor in the implementation of design studies and identifies key barriers to the quick execution of a design study including the identification of and commitment to a domain collaborator, and the mastery of essential domain concepts by the visualization researcher. In our implementation of DSLM, we integrated Service-Learning (S-L) with visualization pedagogy. S-L is an experiential learning model where classroom objectives are aligned with community objectives to meet both educational and community goals. Thus, with this synergy of S-L and DSLM, students are able to quickly "master" the concepts necessary for the design study as most community organizations do not require advanced domain knowledge. It also provides the students with first-hand experience of how their classroom skills can have a positive impact on the community, and the community partners benefit from receiving normally unaffordable data science and visualization expertise.

Hence the contributions of this paper are:

1. A detailed description of DSLM and how it can be incorporated into a typical visualization curriculum to successfully accomplish design studies within 14 weeks.

2. A framework for successfully executing $\mathbf{S}-\mathbf{L}$ in Visualization pedagogy by using DSLM without falling prey to typical design study pitfalls.

3. Reflections on implementations of DSLM, and recommendations for future implementations of the methodology.

\section{RELATED WORK}

In the following section, we review prior work on design studies in visualization and examine relevant works in HCI that have implemented teaching design studies to students. In addition, since we advocate for the application and use of ServiceLearning in the context of adopting DSLM for visualization pedagogy, we will also review prior work done to clearly elucidate the meaning of Service-Learning and pathways for its eventual implementation.
Design Study Methodologies in Visualization: Design studies in visualization are built upon the typical research methodologies in HCI such as open-ended qualitative interviewing $[6,7,8,9,10]$, participatory designing [11], brainstorming of ideas [12], and working together with end users [11, 13]. Domain characterization is the first stage of visualization design. Regardless of the aim of the project, the domain experts' needs, tasks and goals, the conditions under which the visualization will be used, and the constraints on the visualization performance have to be first discussed, characterized, clarified, and sometimes re-scoped $[10,14,15,16]$. Visualization design studies typically involve researchers working alongside domain experts to tackle a real-world challenge, develop a solution and then retrospectively evaluate their work. A seminal work in this space is Munzner's nested model for visualization design [14]. Her model consists of four levels, namely: domain and the characterization of the problem, operation abstraction design, interaction design and finally algorithm design. The nested model provides guidance and recommendations for design practices at each of the levels. Based on this nested model, and the most relevant to our work is the framework for conducting design studies by Sedlmair et al. [1]. The proposed methodological framework consists of 9 stages namely: learn, winnow, cast, discover, design, implement, deploy, reflect, and write. For each stage, the authors provide practical guidance and outline potential pitfalls that serve as checkpoints for the abridged Design Study "Lite" Methodology that we propose in our work.

While relatively less work has been done on design studies themselves, researchers have proposed design 'models' which are frameworks that can be adopted for conducting design studies. For instance, McKenna et al. proposed the Design Activity Framework based on Munzner's nested model, where users Understand, Ideate, Make and Deploy (UIMD) [17]. The model is used as part of a wider project plan with several deadlines throughout the whole project. Lloyd and Dykes looked into problem analysis and paper prototyping in a longitudinal geo-visualization design study, providing interesting insights into which human-centered methods work and which do not [18]. Amar and Stasko provided guidance for bridging identification and communication gaps between the representation and the analysis of data [19]. Van Wijk's model argued for considering the structure and semantics of data in addition to the end user needs through virtue of a visualization value approach [20].

A few other related works also talk about the evaluation of visualization designs and tools in real-world settings [21, 22]. Wang et al. proposed a methodology to introduce data visualization to novice people through interactive workshops in the context of developing comics [23]. The 3-stage explanatory visualization framework guides visualization students to come up with creative yet constrained and domain specific visualizations [24]. Roberts et al. proposed the Five Design Sketches Methodology that walks people through various stages of design development. The model requires users to iteratively sketch their design while contemplating the overall goal of the tool, its operations and interactions. While inspiring our work to an extent, it is also significantly different since the proposed 
method is primarily for the 'ideation stage' rather than the other stages of development [25]. Our work provides a framework through a higher level articulation of methodology. This is significantly different from most of the above work that are excellent resources for conducting more bespoke applications.

The Design Council's double diamond [26] places this in a broader context wherein users upon discovering the problem, define, develop, and deliver the product. This is also seen in Agile Software Development [27], where the approach is to identify which requirements and solutions evolve through the collaborative effort of self-organizing and cross-functional teams and their customer/end user. Further, our approach also fits the Genex framework, which has shown to be effective for successful ideation [28, 29].

Design in HCI pedagogy: Interaction design plays an increasingly prominent role in computing research, while professional user experience roles expand. These forces drive the demand for more design instruction in $\mathrm{HCI}$ classrooms [30, 31]. However, educational research on design in HCI has been limited to a focus on the use of specific techniques or methods such as sketching and storyboarding [32, 33, 34]. Few have focused on pedagogical methods in the HCI classroom, but are not necessarily concerned with design methods. For example, to support student collaboration in HCI group projects, Felker et al. proposed the integration of a scrum software development methodology with user experience development method [35]. Plimmer et al. emphasized the use of peer learning in a multidisciplinary group setting [36] while others have advocated for the use of case studies in the classroom [30, 37, 38]. Within visualization itself, Roberts et al. proposed an explanatory visualization framework that can be used to teach strategies for the implementation of more effective visual designs for learners in higher education [24]. Research has highlighted significant issues in bringing design education to HCI students, including the incorporation of project-based curriculum $[39,40]$. These concerns have continued to exist in today's HCI classrooms, motivating research efforts focused on creating curricula that support successful integration of creative practices with traditional educational methods [37, 41, 42]. Based on these insights, we argue for the need for concentrated research efforts that can inform and shape the HCI design education landscape as our field moves forward and argue for our Design Study "Lite" Methodology that successfully integrates teaching design studies in visualization pedagogy, while offering mutual benefits to students and real-world partners.

Service-Learning (S-L): The proposed framework is evaluated in the context of Service-Learning. Since we also argue for Service-Learning to be used as a key application of DSLM at later stages of this work, we would like to introduce the term here and cite a case study that has successfully applied the proposed methodology in this domain [43].

Service-Learning is an experiential learning model in which classroom learning objectives are aligned with community service to meet both educational and community goals $[44,45$, 46]. There are various methods of Service-Learning including volunteerism, community service, internships, and field education [44]. A key component of Service-Learning is reflection either through written prose or group discussion in order for the student to develop critical thinking skills as well as reflect on how their learning and service relate [47]. Many positive effects of Service-Learning on students have been demonstrated including the development of a sense of meaning and purpose to their academic studies, interpersonal and communication skills, and leadership skills. Service-Learning has also been shown to foster diversity awareness, exposure to other cultures, and an increased sense of community.

\section{DESIGN STUDY "LITE" METHODOLOGY (DSLM)}

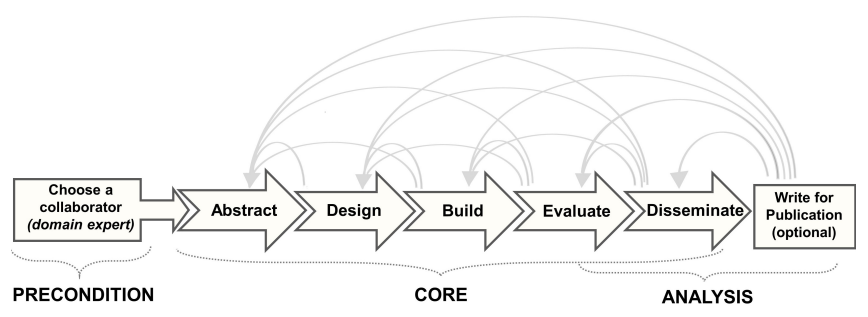

Figure 2. The Design Study "Lite" Methodology framework. This was inspired by the framework of Sedlmair et al. [1] for design studies.

The Design Study "Lite" Methodology (DSLM) is an abridged version of the nine-stage design study framework proposed by Sedlmair et al. [1]. While typical design studies take months to even years for implementation, DSLM enables an expedited project timeline. Following the nested model by Munzner [14] and with additional preconditions and scoping, DSLM is a framework for achieving a complete design study in just 14 weeks. DSLM consists of the essential components of a design study including, interview of a domain expert/stakeholder, iterative design ideation, development of a final product, evaluation of the prototype through user studies followed by summarising the findings by writing a final report and handover of the product to the stakeholders. Further, DSLM also adapts Sedlmair's nine-stage framework [1] (Figures 4(b), 6(b), 7(b), 8(b), 9(b), 10(b) and 11(b)) by truncating the precondition stage and shifting it prior to the beginning of the project. In the DSLM implementation in a standard visualization curriculum, the precondition stage is completed by the instructor before the start of the semester. In addition, while the ninestage framework [1] constitutes a writing for publication stage, in DSLM, students are only required to have a first pass at the writing. However, the students are still required to submit a conference style report as a part of their final submission.

Our Design Study "Lite" Methodology comprises of 7 stages: (1) Choose a collaborator is the first stage where a suitable domain partner is identified. (2) Abstract the key tasks and questions to answer through the visualization, by observing and interviewing domain partners. (3) Design a data representation for the tasks through an iterative process with the domain partners. (4) Build a solution as a usable tool or pipeline for the domain partners. (5) Evaluate the solution through user studies to understand its utility and effectiveness. (6) Disseminate the final product and share the study results. (7) Write for publication is an optional step and can be carried out if the students are interested. 


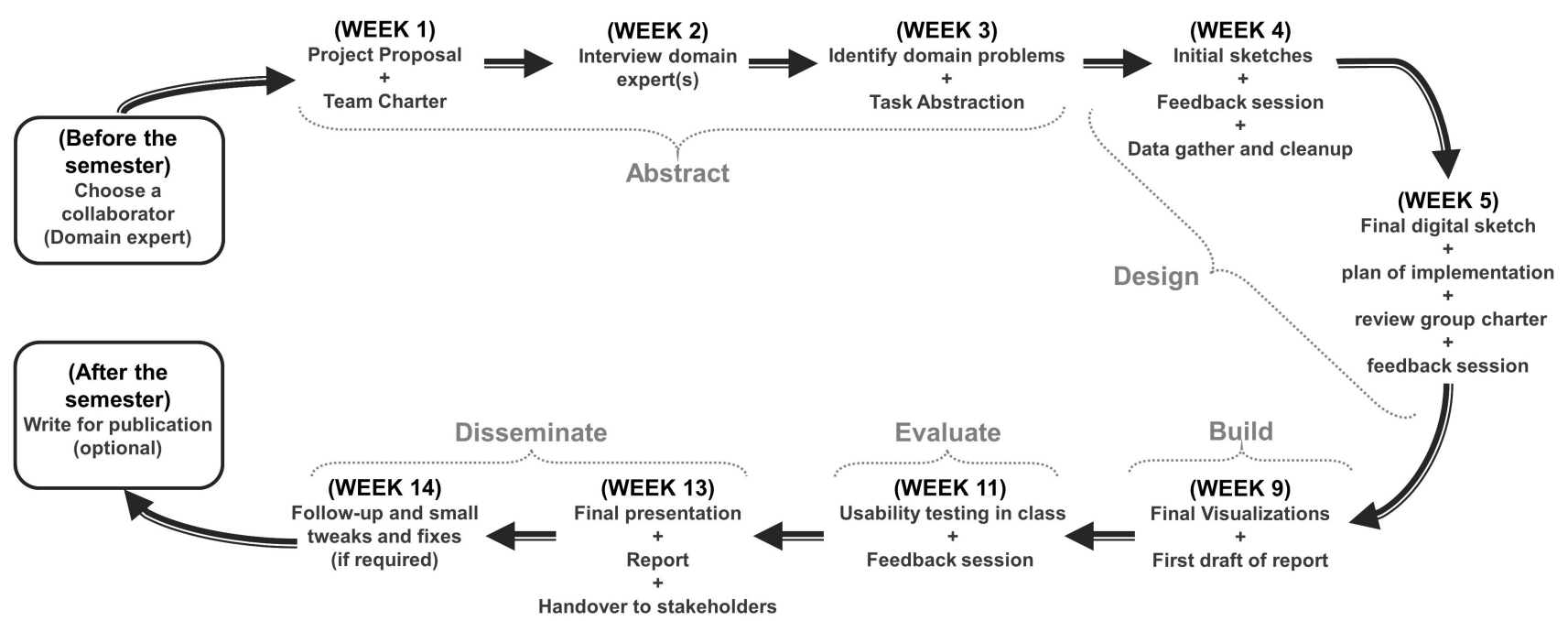

Figure 3. The timeline of Design Study "Lite" Methodology demonstrating how it can be implemented in a semester. The weeks indicate the end of the week i.e when the corresponding work is due. For example, by the end of week 5 , the final digital sketch should be ready and then from the end of week 5 to the end of week 9 , the final visualizations and the report draft should be ready.

In order to expedite the process to fit the duration of a semester, the following modifications and specifications to the design study methodology were applied for the course curriculum:

Choose a domain problem that is easy to understand. When most visualization practitioners apply the design study methodology to a project with domain experts, the required prerequisite knowledge and skills necessary for effectively understanding the domain may require extensive background reading or training with experts. This level of expertise is unattainable in just a couple weeks. To minimize this information and skill acquisition hurdle, community partners and associated questions were chosen to be as easy to understand to a non-expert as possible. This was relatively easy when recruiting community partners as most non-profit community organizations focus on relatable topics such as education, volunteerism, neighborhood improvement, etc.

Work with pre-curated and "clean" data. A pervasive component of the visualization research process is cleaning, analyzing, and mining data. Although the latter two concepts are important to a visualization-focused data science course and enable students to gain experience with exploratory visualizations, data cleaning can be an arduous long iterative process. In order to minimize the time spent on data clean-up, precurated relatively clean data sets were solicited from partner organizations. Note that even with "clean" data, the students still had to clean the data and apply data standardization strategies - a valuable learning experience without distracting the student from the main visualization focus of the assignment.

Structure and limit the amount of iteration. A key part of the design study methodology is the iterative process but this cyclical process can take a very long time. Hence to provide iteration, but limit its duration, a pre-defined number of feedback sessions with the domain experts was set at the beginning of the project. Although this may hamper the full potential of the final product, the compromise is an expedited timeframe. For the course the primary text utilized was Munzner's "Visualization Analysis and Design" [48] which in addition to covering much of the visualization theory and concepts in the course curriculum, is naturally structured to be complementary to teaching the design methodology to students.

\section{IMPLEMENTATION OF DESIGN STUDY "LITE" METHOD- OLOGY IN A SEMESTER}

In this section, we describe the steps of DSLM in more detail in the context of visualization pedagogy and Service-Learning, and explain how each step is in coherence with the original nine-stage design study methodology [1] by showing both frameworks side-by-side for clarification. We also mention the learning objectives ("LOs") for the students for each step of DSLM. The high level LO of DSLM is to learn how to conduct design studies for real world domain experts.

\section{Choose a collaborator Stage}

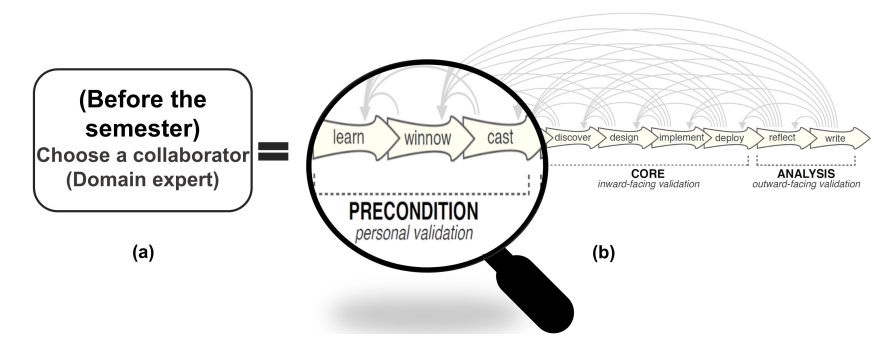

Figure 4. The choose a collaborator stage of DSLM in (a) is equivalent to the Precondition stage in the nine-stage framework shown in (b) [1].

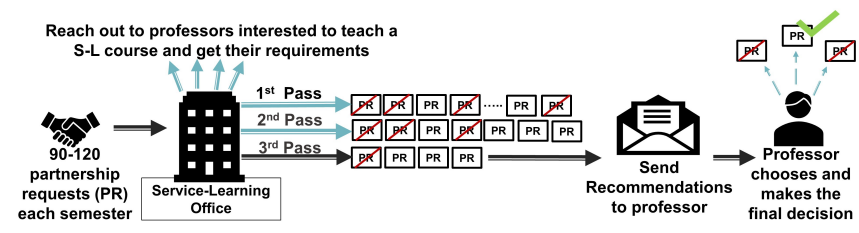

Figure 5. Choosing a community collaborator process using a ServiceLearning facility.

In DSLM with S-L, a community partner is chosen by the instructor via their own connections or through the support of 
a Service-Learning facility within the institution. We advocate for using a Service-Learning facility, as it simplifies the process of identifying the partners. For instance, the ServiceLearning department at Northeastern university has partnerships with over 90-120 non-profit organizations. They identify and recommend suitable projects to instructors after a three step filtering process. Instructors then choose a subset of this list for students to work on during the course of the semester. This process of choosing a partner starts in January for the fall semester and October for the Spring semester. The process is illustrated in Figure 5 and further explained in the supplemental material of DSLM.

\section{Abstract Stage}

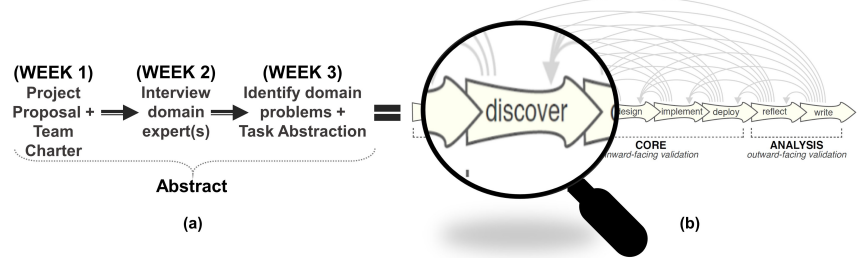

Figure 6. The abstract stage in DSLM (a) is equivalent to the discover stage in the nine-stage framework in (b) [1].

In the abstract stage, the domain problem is identified and translated into tasks. The students are required to prepare a project proposal outlining the domain problem they will tackle and a team-charter that clarifies individual tasks within the team. The student-authored Team Charter ensures that everyone in the group is in sync and understands what they expect of each other. Students compose an agreed-upon team purpose, lay ground rules, and divide responsibilities related to communication, development, and organization of work. The students abstract and understand the domain problem by analyzing data obtained from semi-structured interviews conducted with the community partners. In addition, every student is also required to engage in community service with the partner organization, and reflect on how their volunteering experiences might relate to their final project. Thus, besides doing a comprehensive task analysis, students also get a first hand experience of working for the community. The interview in conjunction with the volunteer experience enable the students to produce a detailed task characterization for their project by the end of week 3 . The students apply the task typology of Brehmer and Munzner [49], and supplement it with the analytic task taxonomy of Amar et al. [19] for finer grain description of low-level tasks. The learning objectives (LOs) of the abstract stage are to learn how to conduct semi-structured interviews, translate domain problems to abstract tasks (task characterization) and to appreciate how data visualization can be used to have a positive impact in the community.

\section{Design Stage}

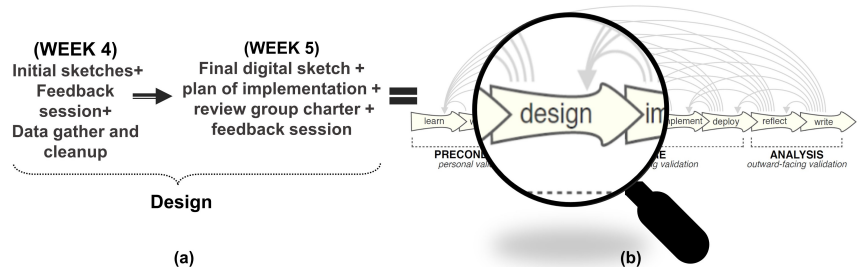

Figure 7. The design stage of DSLM(a) is equivalent to the design stage in the nine-stage framework (b) [1].
In the design stage, the students first clean and mine the data provided by their partner organizations. Then, they generate exploratory visualizations with a standard visualization software, to gain a better understanding of their data and decide on their final visual encoding. Subsequently, the students are required to create multiple hand-drawn sketches and storyboards for the tasks they identified in the Abstract Stage. Each student in a group creates 3 sketches independently and then the group collectively selects the best 3 sketches for their tasks. Every project group then meet with the instructor and Teaching Assistants ("TAs") for feedback on all of their sketches. In addition, they also solicit feedback from their respective partner organizations on the design choices. Once the sketches are finalized, the teams are then required to create a static version of their final visualization with a standard drawing tool. DSLM allocates 2 weeks for this whole process. Choosing appropriate visualization methods for a given data type, designing an effective visualization using design and human perception principles, creating visualizations in Excel, Tableau etc, and critiquing the effectiveness of a visualization design are the LOs of this stage.

\section{Build Stage}

In the build stage, the students create their final web-based interactive visualizations. To demonstrate mastery of key concepts taught in the course, each final design study project visualization is required to include the following: a minimum of two different visual encoding techniques, effective and appropriate use of the color channel for encoding data, brushing and linking interactivity, and details-on-demand interactivity. Students are given 4 weeks to build their final visualizations and are also required to solicit the feedback of the instructor and the TAs and seek assistance from them if required. Besides creating the final visualization(s), they are also required to write a detailed outline of their report in a formal conference-style format.The LOs of the build stage is to implement an interactive web-based visualization that enables data exploration using JavaScript/HTML/CSS and/or Python and to familiarize oneself with the format of a conference style design study paper.

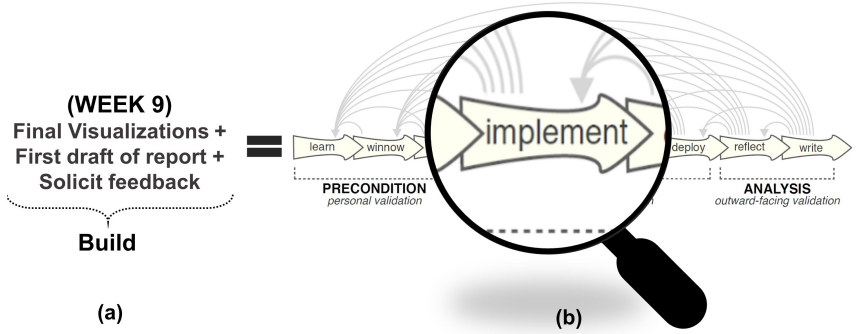

Figure 8. The build stage in DSLM (a) is equivalent to the implement stage in the nine-stage framework (b) [1].

\section{Evaluate Stage}

In the evaluation step, the students continue to refine their visualizations and prepare it for a usability study in class. A full-scale evaluation is not feasible to complete within the time-frame of the semester, so a qualitative usability study is performed by each team to improve their visualization as well as evaluate its effectiveness. The project groups are also required to reflect on the usability testing. Being able to 
constructively critique visualizations, assess its quality and effectiveness and to reflect on one's own design based on user experience and enhancing it are the learning objectives here.

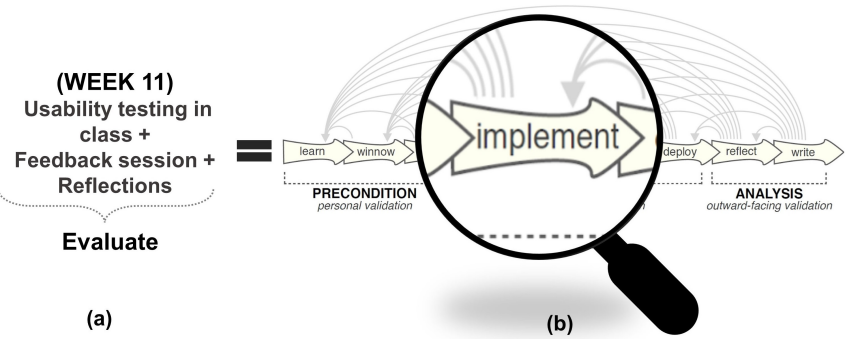

Figure 9. The evaluate stage in DSLM (a) is equivalent to the implement stage in the nine-stage framework shown in (b). [1].

\section{Dissemination Stage}

Every project group is required to create a website with an embedded version of the final visualization(s), and present their final project to the entire class, share the websites with their stakeholders and solicit feedback. The presentations and websites are graded and incorporated into their final design study project grade. All of the community partner organizations are invited to attend the presentations. The students then participate in the Service-Learning Expo, a student poster and demo-fest organized by the Service-Learning Program to present their work. The Expo provides the students the opportunity to further share their project findings and designs with the general public. The LOs in this stage are to create a website and to effectively communicate technical material in oral presentation form.

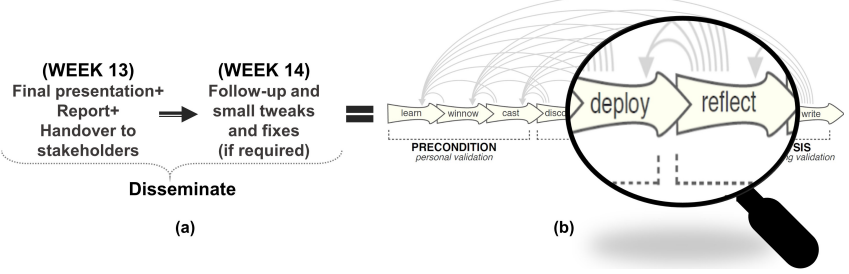

Figure 10. The disseminate stage in DSLM (a) is equivalent to the deploy and reflect stage in the nine-stage framework shown in (b) [1].

\section{Writing Stage}

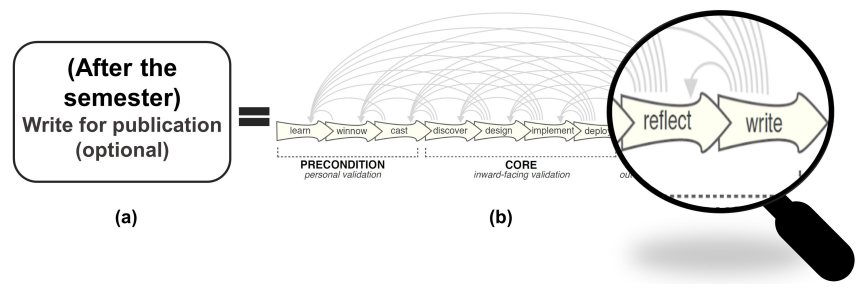

Figure 11. The write stage in DSLM (a) is equivalent to the write and reflect stages in the nine-stage framework shown in (b)[1].

We have seen before that in the "Build" stage, at the end of week 5, students start working of their report. The paper or the final design study project report is for the purpose of getting a good grade. However, students can still write the design study paper for publication [1] as it is included as an optional step in DSLM. We argue that the integration of DSLM in visualization pedagogy also greatly reduces the chances of "writing stage pitfalls" from occurring. This is because, the instructor, along with the TAs, grade the report and provide detailed feedback on each part, making it easier for the students to carefully consider the feedback and prepare it for its publication to a formal venue. This was accomplished by one of the graduate project groups in which the students worked with the Chester Square Neighborhood Association in Boston to help them make a data-driven case in front of the city council to bring to attention the problems plaguing their neighborhood park [43].

\section{HANDLING DESIGN STUDY PITFALLS}

Sedlmair et al. identified and reported that typical design studies might run into 32 pitfalls [1]. In the following section, we assert that the systematic layout of DSLM, in particular with our S-L implementation, greatly reduces the chances of 22 of these pitfalls from occurring in the first place. For example, DSLM is immune to pitfall 1 (shown in Fig12), as students cannot jump to another step without completing the previous ones and running it by their instructor and TAs.

\begin{tabular}{|l|l|l|}
\hline PF-1 & premature advance: jumping forward over stages & general \\
\hline PF-2 & premature start: insufficient knowledge of vis literature & learn \\
\hline PF-4 & premature commitment: collaboration with wrong people & winnow \\
\hline PF-5 & no real data available (yet) & winnow \\
\hline PF-6 & no need for visualization: problem can be automated & winnow \\
\hline PF-7 & researcher expertise does not match domain problem & winnow \\
\hline PF-8 & no need for research: engineering vs. research project & winnow \\
\hline PF-9 & no need for change: existing tools are good enough & winnow \\
\hline PF-10 & no real/important/recurring task & winnow \\
\hline PF-11 & no rapport with collaborators & winnow \\
\hline PF-12 & not identifying front line analyst and gatekeeper before start & cast \\
\hline PF-13 & assuming every project will have the same role distribution & cast \\
\hline$\vdots$ & \multicolumn{2}{|l|}{} \\
\hline PF-16 & expecting just talking or fly on wall to work & discover \\
\hline PF-17 & experts focusing on visualization design vs. domain problem & discover \\
\hline PF-18 & learning their problems/language: too little / too much & discover \\
\hline PF-19 & abstraction: too little & design \\
\hline PF-20 & premature design commitment: consideration space too small & design \\
\hline PF-21 & mistaking technique-driven for problem-driven work & design \\
\hline PF-22 & non-rapid prototyping & implement \\
\hline$\vdots$ & & write \\
\hline PF-28 & insufficient writing time built into schedule & write \\
\hline PF-29 & no technique contribution $\neq$ good design study & write \\
\hline PF-30 & too much domain background in paper \\
\hline PF-31 & story told chronologically vs. focus on final results & write \\
\hline
\end{tabular}

Figure 12. Pitfalls identified by Sedlmair et al. [1]. The red ticks represent the pitfalls that DSLM handles through its systematic structure.

Precondition Stage Pitfalls

The process of identifying the S-L partners by the S-L organizers goes through several winnowing stages, so the chances of having premature collaboration are reduced to a great extent(PF-3). Moreover, decisions as to whether it is indeed a domain problem that can benefit from visualization (PF$6,8,9,10)$ and is in the scope of the materials that students will learn in class (PF-7), is taken into account by the instructor while forming these partnerships. Partners also sign a contract to allocate enough time and data for these projects (PF-4,5). The Service-Learning organizers hold several events before, in between and after the semester in order to build rapport and nurture existing relationships that were formed between students, community partners and instructors. Hence, the possibility of not having enough rapport with collaborators (PF-11) is of little concern. Recall that the students are required to submit a team charter specifying which team member will play what role in the project and this team charter is 
also reviewed later down the process to make sure that the role distributions stay unambiguous, thus reducing the possibility of pitfalls 12 and 13.

\section{Core Stage Pitfalls}

The overview of best practices while conducting qualitative interviews by the instructor and presence of TAs during the actual interview ensures that the focus of the interview is to solicit more information about the project requirements and not about the visions of the community organizations (pitfall 17). However, we do observe in our evaluations that some of the student groups fell prey to this pitfall, thus requiring more training and practice on conducting better semi-structured interviews. Regular checkpoints and feedback along this stage with the instructor and TAs ensure that the students are kept on track, while understanding the problem thoroughly, taking care of pitfalls 18, 19, 20 and 22.

\section{Write Stage Pitfalls}

A time-frame of little over two months is given to the students to prepare and submit a formal conference-style report on their design study project. The report is thoroughly reviewed by the instructor and the TAs and they provide a detailed feedback along with the grades guiding the students into writing a good research paper as they know from the review of the instructor on what to improve. Therefore, the chances of running into pitfalls 28,29,30 and 31 are greatly lowered.

\section{EVALUATION AS A TEACHING COMPONENT WITH S-L}

In the following section, we present our evaluation of the DSLM framework, executed in conjunction with our S-L implementation, by assessing the qualitative and quantitative feedback received from both the students and S-L partners. Regarding the prerequisites of these courses, the graduate ones have none other than the ability to code (intermediate grasp on one or more programming languages). The undergraduate courses have a prerequisite of two semesters of introductory programming. In total 66 students did design study projects in the courses across five semesters, out of which 46 students gave feedback on the forms administered by the university's S-L program. Out of the 46 students who provided feedback, 8 students were enrolled in the graduate version of the course while the rest were undergraduate students. Students were asked open response questions on their learning experience in the course, feedback on the instructor, and the community partners during every semester using an online form. Completion of the evaluation form was voluntary.

The lead authors also conducted semi-structured interviews with the courses' community partners to solicit their responses on the course, their experience working with students and their opinions of the final projects. Out of 7 total partners, all provided anecdotal responses to survey questions and 3 partners agreed to an additional in-person interview.

\section{FEEDBACK FROM STUDENTS}

The first and second authors of this work open coded the responses [50] and identified four high-level themes in the student responses using thematic analysis techniques [51].

\section{Better Learning of the curriculum}

Overall, the students felt that they were able to better acclimatize the material learnt in class through its application for developing real-world projects. Some felt that "The service helped in applying course concepts in a real world setting. We were solving real problems using what we learned in class.", while others approved that the "The experience and course concepts were directly linked.". Students also reported about learning to work better in professional settings, something that they do not get in a classroom. "Working with a real client has significantly reinforced this point to me.". Students also felt that the course structure helped them transition from learning information visualization to practicing information visualization in a neat manner with enough practice on "planning and sketching before the actual coding" and "The design process, the process of investigating a new subject-domain, D3-programming, and telling a story."

\section{Impact on the community}

The Service-Learning curriculum also instilled a feeling of giving back to the community in students. Many students could also relate to the sentiment of application of technology to make positive changes in the immediate community: "I definitely want to do some research in this field and possibly continue working with the community and others involved in the project in some capacity." The students also learn how to be better citizens and members of the community, and this experience has instilled in them a sense of ownership and responsibility:"My service has made me interested in the idea of using data science skills to promote social Good." "It helped me see the greater picture of what my skills can be used for." "This allowed me to better understand what living in Boston is like and how I can give back to the city while I am here at Northeastern [University]".

\section{Efficiency in project completion}

Another recurring theme across the feedback from most students was, they felt that the project completion"was never out of reach" and "they could manage their time and energy really well, owing to the structure of the course". Some students also agreed that the periodic checkpoints "motivated them to finish things on time" and "not leave for the last minute like in the past(some of their earlier courses)". Further, students also mentioned about adopting this structure to their research work and projects of other courses. "I think I would probably plan my research going forward, with my advisor", "This model not only gave me good grades, but I never got flustered, so I would use it in some of my other classes too".

\section{Disconnect between S-L Goals and Personal Goals}

However, not all students felt that the Service-Learning component was attuned with their goals and expectations of the course. A few students felt that "A more technical project would have been in sync with what we learnt in the class and hence a better application of the concepts", while others felt that "There was a disconnect between the class's technical background which led to the covered course concepts being largely irrelevant to completion of our projects". In future implementations of the framework, where the design study project is an important component of the course, care should 
be taken when guiding the students along these projects and focus of the curriculum should not be compromised.

Further, five students also argued for better communication protocol between the community partners and themselves, especially in the earlier versions of the course. They expressed that it was hard to get all the information through one round of interviews alone "We had one interview, which was sufficiently uninformative that we then had to talk with the professor to figure out what we were actually supposed to do", and that more checkpoints are required with the community partners "We would have ideally liked to meet them at least once a month". This was taken into account when refining DSLM over the course of different semesters. Students ultimately though acknowledged that the meetings were organized in the best interests of time and that a major takeaway from the course for them is that "I have a better understanding of how to deal with real-world projects" and that they would "carry forward some of the lessons learnt for their own user-centric research work".

\section{Quantitative evaluation of the course}

Students were also asked to rate the instructor, ServiceLearning TA (S-LTA), S-L partner, and the overall course on three different Likert Scale Measures via a survey. All scales were 3 item, 5 point questionnaires. Results for all four evaluations were rated above neutral. The average ratings for the instructor: 4.94, S-LTA: 4.32, S-L Partner: 3.89, and overall course: 4.31 were all significantly greater than the neutral rating. The respective ratings all monotonically increased over time showing that the improvements and modifications made over time to DSLM worked, although there were no significant differences in them primarily owing to our small sample sizes. The average course rating for the first semester of DSLM was 3.50, whereas the average course rating for the most recent version of the course was 4.06. These metrics show the effectiveness of perfecting DSLM as a part of the course over time, while not compromising on the overall objectives of the class.

\section{FEEDBACK FROM THE SERVICE-LEARNING PARTNERS}

In addition to the student evaluation, the community partners were also interviewed about their experiences working with the students, instructors and the overall design of the final product. The final design study project accounted for $40 \%$ of the total grade for the course and the partners' feedback is an important component because it affected the project grade implicitly in each project milestone given to the students. Detailed grading rubrics are provided in the supplemental material. Five of the community partners continued to work with the instructor over different semesters, indicating their satisfaction with the deliverable provided in the previous semesters. This exhibits their willingness to work with the instructor and DSLM again, standing testimonial to the success of the previous groups' works and demonstrating the applicability of DSLM to realworld problems.

\section{Usefulness of the course}

All of the partners who were interviewed agreed that the S-L program was useful and provided them with "treasure trove of data and insights" and that "it helped build the capacity to accomplish organizational goals." Some partners were surprised by the findings and insights brought together by students working on their data, "The students bring great technologies and fresh eyes to our challenges.", "...it brought out trends in our data in ways we had not seen before".

\section{Success of the project}

The partners agreed that the projects were successful due to a variety of reasons such as "being presentable to the community", "quality matches the standards we display on our organization website" and helping them understand "the data we have". Some also attributed the success of the project to getting "students passionate about community service" and "helping students connect with service by use of their technical skills". One particular partner even mentioned that "seeing these students work reminded me of how I got into community service and I wish this course is to them as what my early days into community service was to me".

\section{Learning experience for the partners}

When asked about how they could make the projects more successful, two of the partners mentioned about adapting and tailoring their requirements to the student population "It's $a$ learning experience for us and may be going forward, we can involve the students as well in identifying the goals and objectives", "I would change the goals if the students feel they can do a better job of something else and present a case for it", they also expressed concerns that the students should be given a stronger background knowledge before the semi-structure interviews in order to ask more meaningful question "feel like they should already know and should be more specific and asking more detailed questions and meaningful questions. ".

\section{Graduate student work could become more exploratory}

They also expressed concerns that some of the graduate student groups looked at the work from an exploratory point of view, and while that offered a more detailed understanding of the problem in hand, it offered little to do with the solution: "found that the graduate students were presenting the information in confusing ways. It seemed like a little bit much for like what we were looking at. The data that they gave me, I do not want to say that it was indecipherable but to be honest it wasn't useful to me as it took so much time to look at it and understand it that it was not beneficial.", "It was very good for exploring. Like a child in a garden-like I wanna go this way, I wanna go that way, but not to decide I want to do this". Hence, they mentioned the need for the projects to be "straightforward", "direct and to the point" in order to have the set of requirements fulfilled. One particular case was where both graduate and undergraduate students worked with Boston Latin Academy School to visualize their gender and racial makeup in two different semesters. Even though the graduate students did well in the project and created linked pie and bar charts, the partner felt that it was way too much work to get the grasp of the visualization, let alone explain it to others and preferred the simpler version of it made by the undergraduate students. This could be a potential pitfall that future implementations of DSLM need to take into account.

Overall, both students and partners agreed on the successful integration of design study project in the course with objectives being fulfilled at both the classroom and community levels. 


\section{ADVANTAGES OF SERVICE-LEARNING FOR DSLM}

The symbiotic relationship between Service-Learning partners achieving their goals, and novice students getting to work with real-world data prompts us to vouch for the inclusion of Service-Learning in visualization pedagogy.

Provides real-world data science experience. The experiential learning exposes students to "dirty" data and teaches them how to clean, manage, and mine information. The experience thus helps take concepts out of the sterile classroom environment and makes students apply their skills to real problems. This thought was echoed by multiple students across the semesters: "In the classroom we are often given clean, groomed data to work with, but through service learning I was able to learn how to work with messy, real data that serves a real purpose.".

Provides professional experience for students. Students learn to communicate and work with stakeholders. These invaluable skills prepare students to be responsible, accountable and professional that goes a long way in helping in their future careers. Once again, several students mentioned that "I had a chance to develop professionally and collaborate on a project on a team.", "I understand how to communicate with people in my community more." It also instilled a sense of responsibility in students due to the involvement of multiple stakeholders: "This makes accurately portraying their data even more important to me, because I now know how important they are to the community and how much these visualizations can help them understand where they can improve."

Impact on a personal level. Students also felt that the experience of working with the community and giving back, helped them understand some of the challenges their neighborhoods feel. While some mentioned that they would like to continue working with the community partners, others said that the exposure from the project made them feel more connected to the problems on an individual level. "This experience definitely made the data feel a lot more personal." "The power of being connected to an individual's needs was powerful."

\section{REFLECTIONS AND RECOMMENDATIONS}

The Design Study "Lite" Methodology has been revised and improved over the course of 5 semesters. This longitudinal and iterative process of improvement has ensured that the initial hurdles were addressed in the subsequent design studies. In the following section, we reflect upon our experience of implementing DSLM and provide a retrospective analysis on the lessons learned. We also offer helpful suggestions for future applications of this framework.

Adapting to different semester lengths. DSLM can be adapted to different course durations. In a shorter term of 12 weeks, the time for building visualizations can be reduced from 4 to 2 weeks and the prerequisite could be updated to include more technical requirements. A duration of 16 weeks could lengthen the development to 6 weeks or include more rounds of user evaluation and feedback.

Setting the stage right. For successful implementation of the framework, it is imperative that the right domain collaborator is chosen. Both the visualization instructor and the domain partner should be on the same page with clear goals.

Employ Service-Learning facilities or plan ahead. A supporting factor to the success of the design study projects were the facilities and infrastructure support from the ServiceLearning Program at Northeastern University. The program helped recruit suitable partner organizations, trained and provided a Service-Learning TA ("S-LTA"), administered formal assessment and evaluation throughout the semester of the course, and conducted an end-of-term Service-Learning Expo to showcase the projects. Implementation of DSLM in conjunction with Service-Learning does not require an affiliated Service-Learning facility (i.e., instructor can identify their own community partners), however if available, it expedites the precondition steps. A list of useful Service-Learning resources are provided in the supplemental materials.

Set realistic expectations. Setting realistic expectations is key for both the students and partner organizations. For the partner, it is important for them to understand that although students are committed to creating a final visualization, there could be a mismatch with the level of sophistication drafted in the prototype sketches. Similarly, it is important for the students to understand that these are real stakeholders and they need to have a quality deliverable by the end of the semester. For this purpose, a signed contract agreed upon between the students, instructor, and organization is essential. Another strategy to help mediate the possible failure of a project is to assign at least two project groups to each organization.

Executing the design process in the right way. Once the prerequisites of a beneficial partnership is taken care of, it is important to maintain a few guidelines for a successful project. Since, the course material and concepts fell in perfect synergy for a design study, we did not run into any major problems during the design process. However, learning from our experience, we would still like to impart a few suggestions that enhance the quality of such projects.

Have more tightly defined guidelines. We interviewed 3 domain partners from the past semesters and 2 of them worked with both graduate and undergraduate projects. We observed that the undergraduate projects were liked more by the partners in comparison to the graduate projects. Upon review and reflection of the courses, a major difference was that the undergraduates were given weekly goals, in contrast to the graduate students who had a milestone every few weeks. Thus, more frequent and tightly defined deadlines, and frequent updates on students' progress might aid towards the success of a project.

Define clear Intellectual Property ("IP") and data use guidelines. Part of setting realistic expectations for the projects is to define clear rules and guidelines on IP and data privacy. Each partner organization was instructed to prepare necessary privacy and/or data use consent forms for their final project student groups. The student groups were also required, if it was not explicitly stated in the organization-provided consent form, to clarify with the organization whether it was acceptable to host their final interactive visualization on a publicly accessible GitHub page or whether the students needed 
to have it privately hosted. The instructor in collaboration with department and legal experts assembled a contract that each organization and student group needed to sign and date at the beginning of the semester. The contract states that the students own the IP, but provides the partner organization with a non-exclusive license for the reuse and modification of their code. The organization can then use, including modify, the visualization as per their needs, ensuring fair use and indemnity of the student.

Maintain effective communication. Of utmost importance is clear and consistent communication between the instructor, S-LTA, students, and partner organizations to ensure that the projects stay on track and meet the educational and community organization's goals. We recommend assigning a Communications Director ("CD") within every student group for this purpose. The CD should be the point of contact with the partner organization, S-LTA, and instructor, on all final project related matters. This will ensure streamlined communication between all the stakeholders of the project.

\section{LIMITATIONS AND FUTURE WORK}

A limitation of the current DSLM implementation is the lack of a full-scale evaluation of the developed visualization. While the usability testing at the end of the semester did indeed give suggestions to eliminate the causes of novices' errors as per [52] and exposed students to user study concepts, the participants of the projects were peers and colleagues who may not be reflective of the actual user. There is also insufficient time to conduct a comparison between the new and existing visualizations. In future work and iterations of DSLM, we also hope to ensure to include the LO where the students are taught in detail on how to choose a domain partner, what to look for, and what to avoid. This will give them additional skills necessary to execute design studies independently. We also want to acknowledge the potential for biased selection when identifying community partners. When collaborating with an S-L program to identify partners, an instructor must trust their judgment. It also may be the case that the organizations in most need of an S-L collaboration do not have the connections or resources to even reach out to an S-L or instructor in the first place. In light of these, we believe the involvement of the instructor curtails this to an extent by allowing the instructor to make the final partnership decisions. Further, a single instructor will never have the kind of outreach an S-L program will to identify community partners. Finally, we look forward to DSLM being used outside the classroom for research projects.

DSLM can also be extended to starter research projects, summer internships, or research training exercises. Regarding scalability, DSLM has been implemented and evaluated for class sizes of 30 students or less by the senior author. A course (with the lead author as TA) with 41 undergraduates was taught by a different professor not involved in the research incorporating DSLM in Fall 2019. The results related to student grades are similar to what has been observed in earlier implementations of DSLM. The role of TA has also provided the lead author with deeper insights into how to make the DSLM process run smoother for large classes (40 students). In future work we plan to pilot DSLM in larger (50+) classes. Additionally, although in our work the connection between visualization and social good was enabled through Service-Learning pedagogy, we strongly advocate that the visualization community look to local community partners for their small (or large) research projects. Building off of recent momentum by the community advocating for ethical visualizations and visualization for social good [53, 54, 55], we too advocate for visualization for social good in teaching and research and look forward to exploring further how DSLM and S-L can support both endeavours.

\section{CONCLUSIONS}

In this work, we present the Design Study "Lite" Methodology, a framework for implementing design studies with novice students in 14 weeks. The methodology can be used in visualization pedagogy in conjunction with Service-Learning, to ensure that the visualization education goals are aligned with community service goals. We also present a successful application of the methodology in a visualization course across 5 semesters, in which 66 students worked on design studies, in multiple small groups, with 7 non-profit community organizations. The students' final design study projects produced new interactive visualizations for the community partners which included visualizations of social impact on the greater community, as well as insights into their data for decision-making. Both students and partner organizations provided positive feedback on the experience, and the instructor as well as some of the students are currently volunteering their time to ensure the final visualizations are incorporated into the organizations internal or external websites. Many organizations are also using the final visualizations as part of their annual impact reports to funders, to demonstrate their community impact. The students in the courses were also more dedicated and committed to their final projects, as compared to the instructor's previous teaching experiences, as their projects had real stakeholders and potential to make an impact on the community. The goal of DSLM is to provide a design study experience for students, even if simplified and expose them to the process rather than a lack of design study experience as is the case in current visualization courses (as mentioned in Introduction). Data visualization has the powerful potential to produce social impact and positive change, and we hope the visualization community continues to develop similar research and education models.

\section{ACKNOWLEDGMENTS}

The authors wish to thank Dr. Michael Weintraub for his inspiration and advice on S-L and computer science pedagogy. We thank Prof. Cody Dunne for providing us with materials from the Fall 2019 DS4200 course to include in our supplemental materials, and in reviewing this paper. We also thank Prof. Timothy Bickmore, Alex Ahmed, Sara Di Bartolomeo, Aditeya Pandey, Ameneh Shamekhi, and Everlyne Kimani for reviewing the paper, and Prof. Tamara Munzner for her early encouragement of DSLM. We would also like to extend gratitude to community partners Carol Blair and Ingrid Roche for their continued collaboration and support of this paper. Finally we thank the staff and S-LTAs of the Service-Learning Program at Northeastern University. This work was supported in part by the Khoury College of Computer Sciences at Northeastern University. 


\section{REFERENCES}

[1] Michael Sedlmair, Miriah Meyer, and Tamara Munzner. Design study methodology: Reflections from the trenches and the stacks. IEEE Transactions on Visualization and Computer Graphics, 18(12):2431-2440, 2012.

[2] Michelle A. Borkin, Zirui Yan, Britton Horn, Lisa Roe, and Becca Berkey. Visualization education through social impact: A service-learning approach for visualization pedagogy. Proceedings of the Pedagogy of Data Visualization Workshop (PDVW 2017), IEEE VIS Conference, 2017.

[3] Michael Sedlmair. Visual Analysis of In-Car Communication Networks. PhD thesis, lmu, 2010.

[4] Tamara Munzner, Qiang Kong, Raymond T Ng, Jordan Lee, Janek Klawe, Dragana Radulovic, and Carson K Leung. Visual mining of power sets with large alphabets. Department of Computer Science, The University of British Columbia, 2, 2005.

[5] Miriah Meyer and Jason Dykes. Criteria for rigor in visualization design study. CoRR, abs/1907.08495, 2019.

[6] John Hughes, Val King, Tom Rodden, and Hans Andersen. Moving out from the control room: ethnography in system design. In Proceedings of the 1994 ACM conference on Computer supported cooperative work, pages 429-439. ACM, 1994.

[7] John A Hughes, Jon O'Brien, Tom Rodden, Mark Rouncefield, and Steve Blythin. Designing with ethnography: a presentation framework for design. In Proceedings of the 2nd conference on Designing interactive systems: processes, practices, methods, and techniques, pages 147-158. ACM, 1997.

[8] Andrew Crabtree, Tom Rodden, Peter Tolmie, and Graham Button. Ethnography considered harmful. In Proceedings of the SIGCHI Conference on Human Factors in Computing Systems, pages 879-888. ACM, 2009.

[9] Gillian R Hayes. The relationship of action research to human-computer interaction. ACM Transactions on Computer-Human Interaction (TOCHI), 18(3):15, 2011.

[10] Yvonne Rogers, Helen Sharp, and Jenny Preece. Interaction design-beyond human-computer interaction, 2012.

[11] Richard Wiseman. 59 seconds: think a little, change a lot. Random House Canada, 2010.

[12] Francis Crick. The impact of linus pauling on molecular biology: A reminiscence. The chemical bond: Structure and dynamics, pages 87-98, 1992.

[13] Daniel F Keefe, Daniel Acevedo, Jadrian Miles, Fritz Drury, Sharon M Swartz, and David H Laidlaw. Scientific sketching for collaborative vr visualization design. IEEE Transactions on Visualization and Computer Graphics, 14(4):835-847, 2008.
[14] Tamara Munzner. A nested model for visualization design and validation. IEEE Transactions on Visualization and Computer Graphics, 15(6):921-928, 2009.

[15] G Elisabeta Marai. Activity-centered domain characterization for problem-driven scientific visualization. IEEE Transactions on Visualization and Computer Graphics, 24(1):913-922, 2017.

[16] Don Norman. The design of everyday things: Revised and expanded edition. Basic books, 2013.

[17] Sean McKenna, Dominika Mazur, James Agutter, and Miriah Meyer. Design activity framework for visualization design. IEEE Transactions on Visualization and Computer Graphics, 20(12):2191-2200, 2014.

[18] David Lloyd and Jason Dykes. Human-centered approaches in geovisualization design: Investigating multiple methods through a long-term case study. IEEE Transactions on Visualization and Computer Graphics, 17(12):2498-2507, 2011.

[19] Robert Amar, James Eagan, and John Stasko. Low-level components of analytic activity in information visualization. In IEEE Symposium on Information Visualization, 2005. INFOVIS 2005., pages 111-117. IEEE, 2005.

[20] Jarke J Van Wijk. Views on visualization. IEEE Transactions on Visualization and Computer Graphics, 12(4):421-432, 2006.

[21] Heidi Lam, Enrico Bertini, Petra Isenberg, Catherine Plaisant, and Sheelagh Carpendale. Empirical studies in information visualization: Seven scenarios. IEEE Transactions on Visualization and Computer Graphics, 18(9):1520-1536, 2011.

[22] Sheelagh Carpendale. Evaluating information visualizations. In Information visualization, pages 19-45. Springer, 2008.

[23] Zezhong Wang, Harvey Dingwall, and Benjamin Bach. Teaching data visualization and storytelling with data comic workshops. In Extended Abstracts of the 2019 CHI Conference on Human Factors in Computing Systems, page CS26. ACM, 2019.

[24] Jonathan C Roberts, Panagiotis D Ritsos, James Jackson, and Chris Headleand. The explanatory visualization framework: An active learning framework for teaching creative. IEEE Transactions on Visualization and Computer Graphics, 10.

[25] Jonathan C Roberts. The five design-sheet (fds) approach for sketching information visualization designs. In Eurographics (Education Papers), pages 29-36, 2011.

[26] Design Council. Eleven lessons: Managing design in eleven global companies-desk research report. Design Council, 2007. 
[27] Ken Schwaber and Mike Beedle. Agile software development with Scrum, volume 1. Prentice Hall Upper Saddle River, 2002.

[28] Ben Shneiderman. Creating creativity: user interfaces for supporting innovation. ACM Transactions on Computer-Human Interaction (TOCHI), 7(1):114-138, 2000.

[29] Elizabeth B-N Sanders and Pieter Jan Stappers. Co-creation and the new landscapes of design. Co-design, 4(1):5-18, 2008.

[30] Lauren Wilcox, Betsy DiSalvo, Dick Henneman, and Qiaosi Wang. Design in the hci classroom: Setting a research agenda. In Proceedings of the 2019 on Designing Interactive Systems Conference, pages 871-883. ACM, 2019.

[31] Jonathan Lazar, Jenny Preece, Jean Gasen, Terry Winograd, and Terry Winograd. New issues in teaching hci: pinning a tail on a moving donkey. In $\mathrm{CHI} O 2$ Extended Abstracts on Human Factors in Computing Systems, pages 696-697. ACM, 2002.

[32] Khai N Truong, Gillian R Hayes, and Gregory D Abowd. Storyboarding: an empirical determination of best practices and effective guidelines. In Proceedings of the 6th conference on Designing Interactive systems, pages 12-21. ACM, 2006.

[33] Káthia Marçal de Oliveira, Patrick Girard, Taisa Guidini Gonçalves, Sophie Lepreux, and Christophe Kolski. Teaching task analysis for user interface design: lessons learned from three pilot studies. In Proceedings of the 27th Conference on l'Interaction Homme-Machine, page 31. ACM, 2015.

[34] Rui Chen, Po-Jui Ray Chen, Rui Feng, Yilin Elaine Liu, Andy Wu, and Ali Mazalek. Scisketch: a tabletop collaborative sketching system. In Proceedings of the 8th International Conference on Tangible, Embedded and Embodied Interaction, pages 247-250. ACM, 2014.

[35] Chase Felker, Radka Slamova, and Janet Davis. Integrating ux with scrum in an undergraduate software development project. In Proceedings of the 43rd ACM Technical Symposium on Computer Science Education, pages 301-306. ACM, 2012.

[36] Beryl Plimmer and Robert Amor. Peer teaching extends hci learning. In ACM Sigcse Bulletin, volume 38, pages 53-57. ACM, 2006.

[37] Mary Beth Rosson, John M Carroll, and Con M Rodi. Case studies for teaching usability engineering. In $A C M$ SIGCSE Bulletin, volume 36, pages 36-40. ACM, 2004.

[38] D Scott McCrickard, Christa M Chewar, and Jacob Somervell. Design, science, and engineering topics?: teaching hci with a unified method. In ACM SIGCSE Bulletin, volume 36, pages 31-35. ACM, 2004.

[39] Xristine Faulkner and Fintan Culwin. Enter the usability engineer: integrating hci and software engineering. In ACM SIGCSE Bulletin, volume 32, pages 61-64. ACM, 2000.
[40] Eli Blevis, Yvonne Rogers, Martin Siegel, William Hazlewood, and Amanda Stephano. Integrating hci and design: Hci/d at iub, a design education case story. In Zimmerman, J., Evenson, S., Baumann, K., \& Purgathofer, $P$. Workshop on the relationship between design and HCI. ACM CHI 2004 Conference on Human Factors and Computing Systems. Vienna, Austria. http://k2. iguw. tuwien. ac. at, volume 8080, 2004.

[41] Mihaela Vorvoreanu, Colin M Gray, Paul Parsons, and Nancy Rasche. Advancing ux education: A model for integrated studio pedagogy. In Proceedings of the 2017 CHI Conference on Human Factors in Computing Systems, pages 1441-1446. ACM, 2017.

[42] Piotr D Adamczyk and Michael B Twidale. Supporting multidisciplinary collaboration: requirements from novel hci education. In Proceedings of the SIGCHI Conference on Human Factors in Computing Systems, pages 1073-1076. ACM, 2007.

[43] Uzma Haque Syeda, Prasanth Murali, and Michelle Borkin. Chester square park: A case study of visualization for social good using design study "lite" methodology. In Posters presented at the IEEE Conference on Visualization (IEEE VIS 2019), Vancouver, Canada, October, 2019.

[44] Andrew Furco. Service-learning: A balanced approach to experiential education. 1996.

[45] Barbara Jacoby et al. Service-Learning in Higher Education: Concepts and Practices. The Jossey-Bass Higher and Adult Education Series. ERIC, 1996.

[46] Robert Sigmon. Service-learning: Three principles. Synergist, 8(1):9-11, 1979.

[47] Janet Eyler and Dwight E Giles Jr. Where's the Learning in Service-Learning? Jossey-Bass Higher and Adult Education Series. ERIC, 1999.

[48] Tamara Munzner. Visualization analysis and design. A $K$ Peters Visualization Series, CRC Press, 2014.

[49] Matthew Brehmer and Tamara Munzner. A multi-level typology of abstract visualization tasks. IEEE Transactions on Visualization and Computer Graphics, 19(12):2376-2385, 2013.

[50] Hsiu-Fang Hsieh and Sarah E Shannon. Three approaches to qualitative content analysis. Qualitative health research, 15(9):1277-1288, 2005.

[51] Virginia Braun and Victoria Clarke. Using thematic analysis in psychology. Qualitative research in psychology, 3(2):77-101, 2006.

[52] Valerie Mendoza and David G Novick. Usability over time. In Proceedings of the 23rd annual International Conference on Design of Communication: Documenting \& Designing for Pervasive Information, pages 151-158. ACM, 2005. 
[53] Michael Correll. Ethical dimensions of visualization research. In Proceedings of the 2019 CHI Conference on Human Factors in Computing Systems, page 188. ACM, 2019.

[54] Evan M Peck, Sofia E Ayuso, and Omar El-Etr. Data is personal: Attitudes and perceptions of data visualization in rural pennsylvania. In Proceedings of the 2019 CHI Conference on Human Factors in Computing Systems, page 244. ACM, 2019. 